\title{
Bioprocessing and Tissue Interactions of Elongated Mineral Particles, EMP, Following Inhalation Exposure
}

\author{
Uschi Graham $^{1}$, Alan Dozier ${ }^{1}$, Chen Wang ${ }^{1}$ and Joe Fernback ${ }^{1}$ \\ 1. National Institute of Occupational Safety and Health, Cincinnati, $\mathrm{OH}$.
}

Preliminary results from examining lung tissue from a former asbestos miner in Quebec are presented. Asbestos, silica, and other types of EMP's are considered to be very bio-persistent. Toxicological studies of inhaled metal-oxide nanoparticles show unexpected chemical and structural bioprocessing even for highly insoluble bio-persistent materials ${ }^{1,2,3}$. This is what motivated this exploratory work. This study utilized a JEOL 2100F high resolution analytical transmission electron microscope (HRTEM/STEM) with sub-nanometer resolution, coupled with EELS and EDS detectors. We report the ultrastructural characteristics for several EMP's, detailing chemical concentration gradients inside an iron-protein coating, and also lateral elemental gradients in the local tissue region. These observations suggest that EMP's and possibly asbestos fibers are subjected to bioprocessing which involves physicochemical transformations and elemental transport mechanisms that alter the inhaled EMP as well as the surrounding cellular matrix. We show at high resolution a ferruginous fiber coating around an EMP is observed to have a distinct nanostructure with some parallel aligned nano-fibrils which are to some extent reminiscent of natural tooth enamel which contains bio-mineralized nanocomposites with alternating organic/inorganic matrices in aligned channels. These are known for their phenomenal strength as well as durability. Future study of these coatings may shed light on variations in the persistence of different types of EMP's.

Figure 1 shows an asbestos like EMP with copious amounts of ferritin particles in its vicinity. Increased ferritin is associated with inflammation. The Figure 1 far right image suggests mass loss in an EMP and possible alteration products in the lower left. These are the first observations and much work remains to be done to fully understand and verify these results. Figure 2 shows several EDS maps of an asbestos like EMP. The iron and magnesium maps suggest diffusion from a silicon based fiber. Prior work has shown that diffusion products can precipitate into 1 to $2 \mathrm{~nm}$ particles ${ }^{3}$. Nanoparticles of this size can be highly reactive. Thus, it would be worth looking for an association between bioprocessing particle production, EMP toxicity, and health effects.

We have shown that the combination of high resolution imaging combined with elemental mapping on select EMP's is a method for evaluating EMP stability versus degree of bioprocessing. It can also be used to analyze the type of processes occurring and products, if any are produced. Further work is required to verify the consistency of the observations here. If silicon based EMP's are subject to long term bioprocessing then the processes need to be determined along with the long term health implications.

\section{References:}

[1] Graham, U.M. et al, Adv Exp Med Biol 947 (2017), p. 71.

[2] Graham, U.M. et al, Proc Micros Micro, St. Louis (2017).

[3] Graham, U.M. et al, ChemPlusChem 79 (2014), p. 1083. 


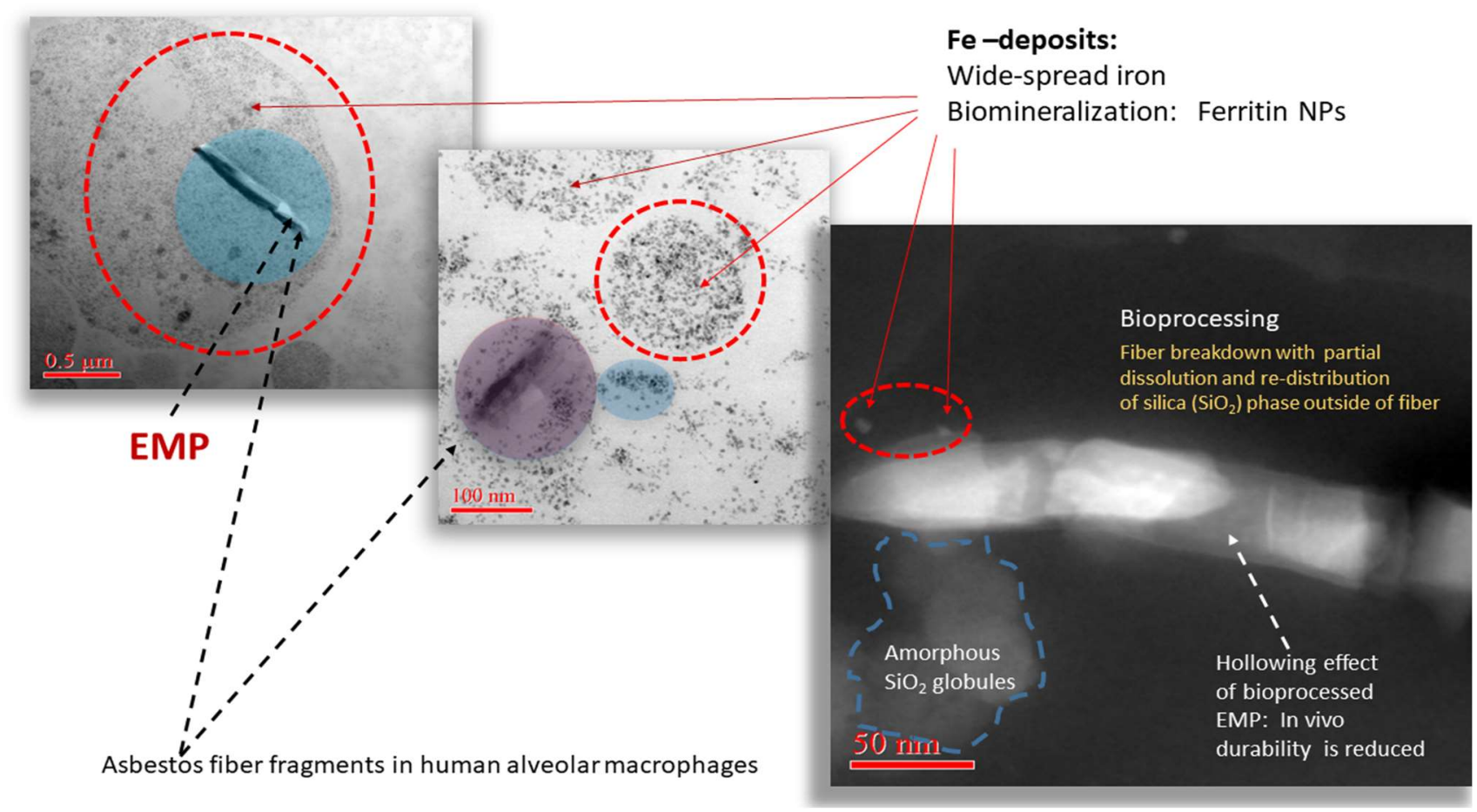

Figure 1. TEM and STEM imaging of Fe-deposits in vicinity of EMP. The Fe-deposits are highly dispersed as clouds. The individual size of individual biomineralized Fe-nanoparticles (ferritin) is $5 \mathrm{~nm}$.
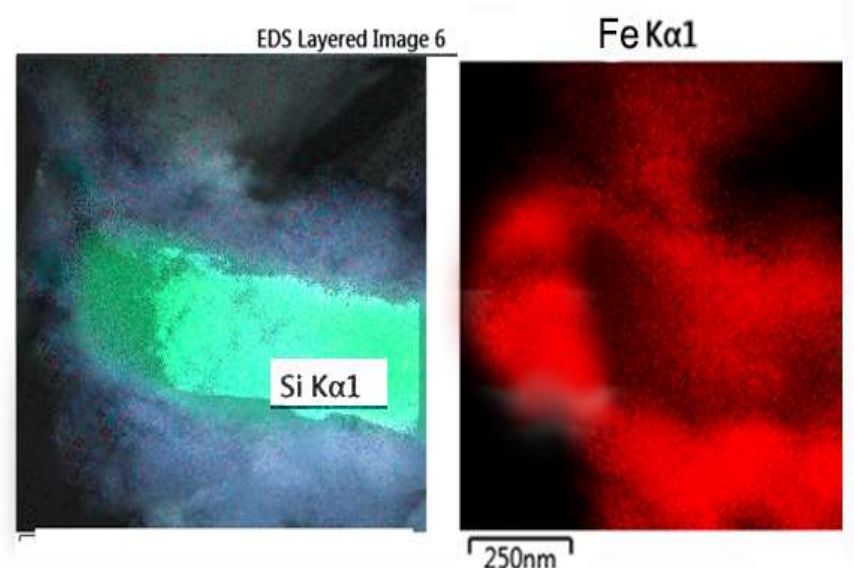

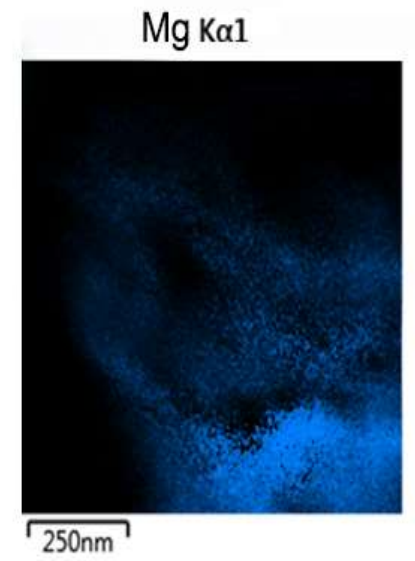

Figure 2. Elemental mapping of ferruginous body with a preserved Si-rich core (left) and a crust of Fe. Carbon enrichment is shown to follow the Fe-rich crust. $\mathrm{Mg}$ is somewhat depleted in the inner core, but much less concentrated in the crust compared with Fe and $\mathrm{C}$. 